\title{
Miniaturized Devices For Au Nanorods Detection
}

\author{
N. Carmona ${ }^{\mathrm{a}}$, V. Bouzas ${ }^{\mathrm{a}}$, J. Jiménez de la Morena ${ }^{\mathrm{b}}$, M. A. García ${ }^{\mathrm{a}, \mathrm{b}}$ \\ ${ }^{a}$ Dept. Fis. Mat. Univ. Complutense Madrid \\ ${ }^{b}$ Instituto de Cerámica y Vidrio, CSIC
}

\begin{abstract}
We present here some miniaturised devices designed for the detection of nanorods in water and blood media. The devices use light emitting diodes as monochromatic light sources and Si photodiodes for detection. Devices working on transmission mode are able to detect nanorods concentration below micrograms/litre. Sensitivity is reduced is one or of magnitude for nanorods in blood. We show detection of low nanorods concentration with devices working in reflection mode, opening the possibility to perform direct imaging of nanorods in vivo. The optima condition for detection and resolution limits are also discussed.
\end{abstract}

Keywords: Nanorods, Surface plasmons, Biophysics

\section{INTRODUCTION}

Gold nanoparticles exhibit an intense absorption band associated with the excitation of surface plasmon resonance (SPR) that allows detecting small amounts of nanoparticles (even single nanoparticles in optima conditions). This absorption can be tuned through the particle geometry. Non spherical nanoparticles as nanorods shows two absorption bands (associated with longitudinal and transversal surface plasmons). One of these bands falls in the IR part of the spectrum where blood and biological tissue scarcely absorbs open the possibility to detect nanorods in vivo. A proper control of the nanorods geometry allows tuning the optical properties of the nanorods to make it detectable in the bloodstream. Actually detection of gold nanorods in bloodstream with a spectrophotometer is well established. A key step toward the application of nanorods for in vivo applications is the development of small devices to interact with the nanorods without the geometrical limitation of laboratory spectrophotometers. To this purpose, miniaturised devices are required to image or energize the nanorods locally. We present here some miniaturised devices designed for the detection of nanorods in water and blood media. The devices use light emitting diodes as monochromatic light sources and Si photodiodes for detection. 


\section{EXPERIMENTAL}

Gold nanorods were synthesised by a seed mediated growth method as described in [1]. Syntehsis parameters were fitted to optimize the optical absorption at the NIR region $\mathrm{f}$ the spectrum. Optical absorption (OA) spectra were recorded by means of a double-beam Shimazu 3101 spectrophotometer attached with an integrating sphere and a reflectance accessory. Liquid samples were placed into a quartz cuvette with an empty cuvette in the reference beampath to remove any contribution to the measurements of the container

\section{RESULTS AND DISCUSSION}

Figure 1a present the optical absorption spectrum of the nanorods in water. Two absorption bands are clearly observed one at about $540 \mathrm{~nm}$ and other at $800 \mathrm{~nm}$ corresponding to the transversal and longitudinal SPR respectively. The spectra of human blood and blood containing a concentration of $1 \mathrm{mg} / \mathrm{l}$ of $\mathrm{Au}$ nanorods is presented in figure 1b. While the transversal SPR band can not be distinguished because of the intense absorption of the blood in the green part of the spectrum (due to the absorption of haemoglobin), the SPR band of longitudinal SPR is clearly resolved confirming the possibility to detect Au nanorods in the bloodstream.
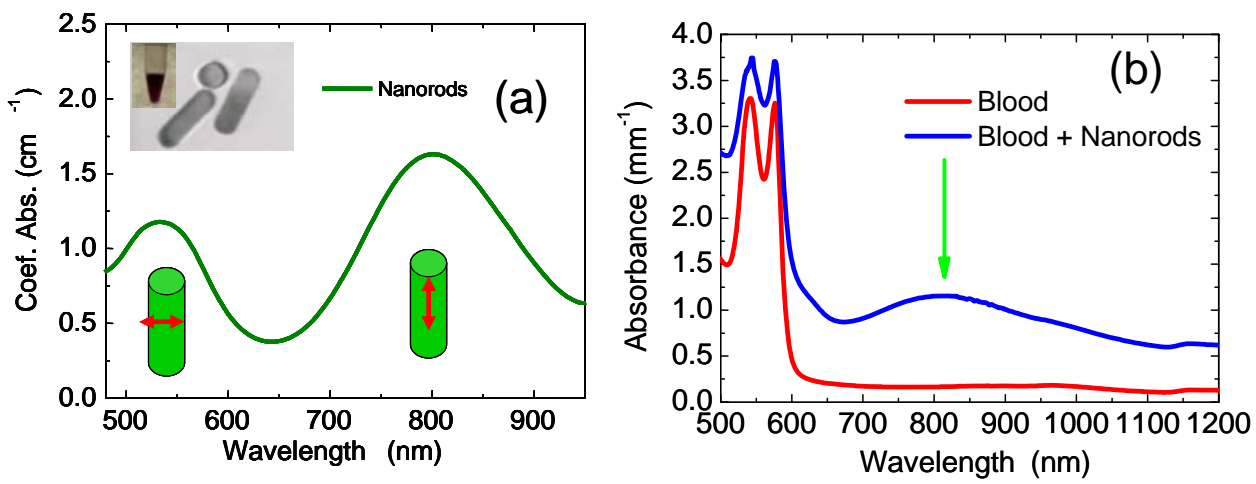

Figure 1. (a) Optical absorption spectrum of Au nanorods with the bands associated to the longitudinal and transversal SPR. Inset shows a TEM micrographs confirming the elongated shape. (b) Optical absorption spectra of human blood and blood containing $1 \mathrm{mg} / \mathrm{l}$ nanorods. To make both curves comparables, the spectrum corresponding to the blood was obtained adding the same amount of water that was added to solve the nanorods in the blood.

Two miniaturised devices to detect nanorods were designed. The first prototype working on transmission mode is presented in figure 2a.

The system uses a LED working at $780 \mathrm{~nm}$ as light source. This wavelength is at about the maxim of the longitudinal SPR band according to figure 1. A Si photodiode with a good sensitivity at this region of the spectrum was used to detect the light. Bias 
voltage was $1.7 \mathrm{~V}$ for the LED and 10 volts for the photodiode. Therefore no high voltages are required to operate the devices avoiding electrical risks for in vivo applications. The LED and Photodiode were powered with standard lab sources. Current flowing through the photodiode circuit was measured with a multimeter by recording the voltage across a resistance in serie. A mechanical device shown in the figure was fabricated. The system consists on a PVC piece with a rectangular cavity to place the cuvette with the sample (figure $2 \mathrm{~b}$ ). A perpendicular path to place the LED and the Photodiode crossing the sample were done. Actually we fabricated two paths in order to have a reference channel if required. An additional slot to place an optical chopper was included in the piece.

The calibration curve for the device is presented in figure 2c.Deviations from linearity are due to errors in measuring the nanorods concentrations in the blood during the experiments but the measurements on a single sample we reproducible with an accuracy of more than $0.01 \%$. From the slope of calibration curve and assuming that values of 1 microvolts can be detected easily with standard electronics the detection limit of the device is of the order of $10 \mathrm{ng} / \mathrm{l}$. When measuring Au nanorods in blood the electrical signals are quite reduced due to the large scattering of blood. Thus, designs working on transmission mode require a short optical path between light source and detector to minimize the effect of scattering. For Au nanorods in blood our detection limit was between 100 and $1000 \mathrm{ng} / \mathrm{l}$.
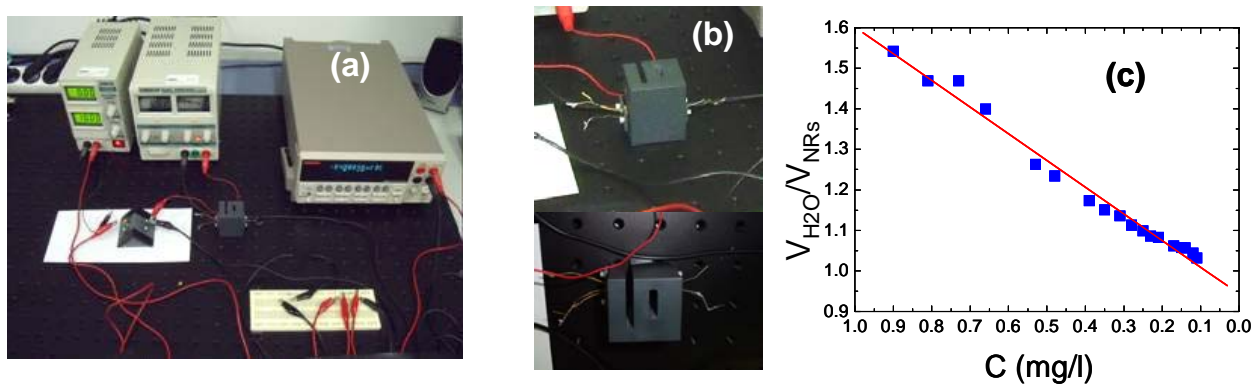

Figure 2. (a) Experimental device for the nanorods detection. (b) Detail of the transmission mode device. (c) Calibration curve for the nanorods in water (photodiode signal detected for the nanorods divided by that of pure water as a reference).

We developed a second prototype working on reflection mode. Reflection is always worse than transmission mode in order to quantify the nanorods concentration. However, in vivo imaging is commonly performed in reflection mode so a reflection device can provide information about nanorods imaging sensitivity. The prototype is shown in figure 3. The sensor head present a roof shape with the LED and Photdiode placed on the roof. The geometry is designed to have the optical focus at the surface where the piece is placed. Thus, the photodiode receives the direct reflected light at the surface. 

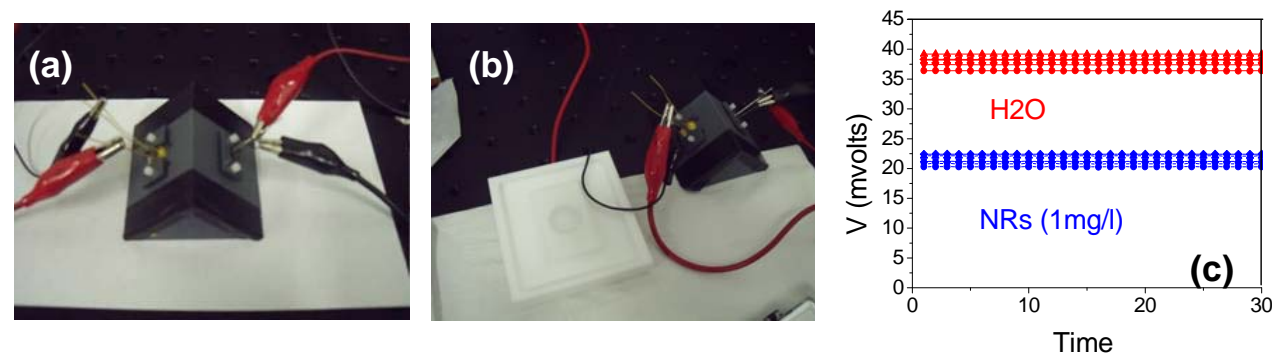

Figure 3. (a) Experimental sensor device for the nanorods detection. (b) detail of the probe head and the sampleholder. (c) Reflectivity measurements for water and water containing nanorods (1 mg/l).

The measurement in reflection mode is highly dependent on the sample position (figure 3c). Small variation in the height of the liquid surface leads to substantial differences in the photodiode signal.. We developed a sampleholder to place the liquid sample shown in figure 3b. Even whit this device, variations of the order of $10 \%$ were observed repeating measurements nominally identical due to positioning differences. Reflectivity depends strongly on the signal at the photodiode and it is not a good geometry to perform quantitative analysis. The interest of reflectivity relays on the fact that images are commonly formed by reflected light and differences in reflectivity depending on the nanorods concentration can allow their imaging by using a IR camera. As shown in figure 3c, nanorods dispersed in water at a concentration of 1 $\mathrm{mg} / \mathrm{l}$ reduce the photodiode signal at about $10 \mathrm{mV}$ and this reduction can not be associated to the sample positioning as evidenced by repeating the measurements several times. The ratio of $10 \mathrm{mV} /(\mathrm{mg} / \mathrm{l})$ is similar to that observed in the transmission device (figure 2) ans suggest that local variation of the order $10 \mathrm{ng} / \mathrm{l}$ can be detected by reflective imaging.

\section{CONCLUSIONS}

Small devices to detect gold nanorods in both transmission and reflection mode. The transmission device allow quantification of nanorods dispersed in water with a resolution of the order of $10 \mathrm{ng} / \mathrm{l}$; and below $1 \mu \mathrm{g} / \mathrm{l}$ for nanorods in blood. The reflection device does not allow quantification of nanorods concentration but can be useful to image the nanorods.

\section{ACKNOWLEDGMENTS}

This work was supported by the STREP FP6 E.U. Project Bio-Imaging with Smart Functional Nanoparticles (BONSAI) Nº LSHB-CT-2006-037639.

\section{REFERENCES}

1. See in this volume Synthesis of Gold Nanorods for Biomedical Applications by N. Carmona et al. 